\title{
The Role of Glycemic Control and Variability in Diabetic Retinopathy
}

Irini P. Chatziralli (D)

Received: October 29, 2017 / Published online: November 30, 2017

(c) The Author(s) 2017. This article is an open access publication

\begin{abstract}
Diabetic retinopathy (DR) is a common microvascular complication of diabetes mellitus (DM) and is considered the leading cause of visual impairment in working-aged adults worldwide. The duration of DM and hyperglycemia have been associated with DR, although the exact role in the pathogenesis of DR and diabetic macular edema remains controversial. As a result, a reasonable question arising is whether control of blood glucose levels may alter the course of DR. Studies have shown that glycemic control remains an important factor for the presence and progression of DR. HbA1c seems to be an indicator which cannot demonstrate exactly the degree of glycemic control, while sudden variations of blood glucose may play an important role in DR; therefore, glycemic variability may be useful to predict DM complications, such as DR.
\end{abstract}

Keywords: Diabetes; Retinopathy; Macular edema; Glucose control; Variability

Enhanced content To view enhanced content for this article go to http://www.medengine.com/Redeem/ 28FCF0606DA5C55D.

I. P. Chatziralli $(\bowtie)$

2nd Department of Ophthalmology, University of Athens, Athens, Greece

e-mail: eirchat@yahoo.gr

\section{COMMENTARY}

Diabetic retinopathy (DR) is a common microvascular complication of diabetes mellitus and is considered the leading cause of visual impairment in adult people of working age in developed countries [1]. DR currently affects almost 100 million people worldwide and is becoming an ever-increasing health burden, with estimates between 1990 and 2010 showing that DR-related visual impairment and blindness increased by $64 \%$ and $27 \%$, respectively [2]. Several risk factors have been associated with DR, while good glycemic and blood pressure control are considered the most important modifiable risk factors to reduce the risk of progression of DR and vision loss [3]. However, recent studies have shown that the improvement in visual acuity and DR severity score after intravitreal anti-vascular endothelial growth factor (anti-VEGF) agents seem to be independent of baseline HbA1c [4]. Therefore, a reasonable question arising is whether glycemic control may alter the course of DR. This article is based on previously conducted studies and does not involve any new studies of human or animal subjects performed by the author.

Two landmark clinical trials, the Diabetes Control and Complications Trial (DCCT) and the United Kingdom Prospective Diabetes Study (UKPDS), have demonstrated the beneficial effects of intensive glycemic control in patients 
with type 1 and type 2 diabetes mellitus, respectively, with the decrease in the incidence and progression of DR [5, 6]. Specifically, in the DCCT, 1441 patients with diabetes mellitus type 1 were randomized to either "intensive" or "conventional" treatment and followed up for 6.5 years, showing that the "intensive" treatment group (mean HbA1c of $7.2 \%$ ) had a $54 \%$ reduction in the incidence and a $76 \%$ reduction in the progression of DR, compared to the "conventional" group (mean HbA1c of 9.2\%), while earlier tight control seemed to be more beneficial [5]. In their follow-up study, the Epidemiology of Diabetes Interventions and Complications (EDIC), patients from the "intensive" group maintained the reduction in risk for DR progression for 4 years, even though HbA1c levels in the two groups gradually converged [7]. This phenomenon could be described as "metabolic memory", which suggested the longlasting benefit of previously intense glucose control. Accordingly, the UKPDS, which involved 3867 newly diagnosed patients with type 2 diabetes mellitus, revealed that patients with intensive glycemic control with a mean HbA1c of $7.0 \%$ had a $21 \%$ reduction in the progression of DR compared to those with conventional glycemic control (mean HbA1c of 7.9\%) [6]. Furthermore, in the Action to Control Cardiovascular Risk in Diabetes (ACCORD) Trial, patients who had intensive blood sugar control with a target HbA1c level of below 6.0\% presented a significantly lower rate of progression of their DR compared to those with a target HbA1c level of $7.0-7.9 \%$ (7.3\% vs $10.4 \%$ progression) [8]. These findings were confirmed by a Cochrane review on intensive glucose control in patients with type 2 diabetes mellitus [9].

Nevertheless, a disadvantage of tight glycemic control was an early initial worsening in DR status, reported in the DCCT, while hypoglycemic episodes were also common [5]. However, these observations were transient and reversed, while the long-term results were in favor of the "intensive" glycemic control. Therefore, the American Diabetes Association recommend that all patients with diabetes (type 1 and type 2) should strive to maintain HbA1c levels of below $7.0 \%$ to prevent or at least minimize the long-term complications of diabetes mellitus, including DR [10].

Although the majority of studies have used $\mathrm{HbA1c}$ as an indicator of the degree of glycemic control, recently attention was paid to glycemic variability $(\mathrm{GV})$, which refers to the oscillations in blood glucose throughout the day, including hypoglycemic periods and postprandial increases, as well as blood glucose fluctuations that occur at the same time on different days. In fact, HbA1c has major limitations and even in the best of circumstances provides only a simplified snapshot of glycemic control. On the other hand, glucose fluctuations have been proven to show a more specific triggering effect on oxidative stress than chronic sustained hyperglycemia does [11].

Previous studies have shown that fasting plasma glucose fluctuation as well as marked daily postprandial GV was associated with diabetes-related cardiovascular diseases [12]. As far as DR is concerned, in patients with type 1 diabetes mellitus, Picconi et al. have shown that early structural damage of neuroretinal cells was related to glucose fluctuation and suggested that GV should be addressed even in the presence of good metabolic control [13], in line with Virk et al., who found that greater HbA1c variability was a predictor for DR [14]. On the other hand, the DCCT group suggested that within-day GV does not play an apparent role in the development of microvascular complications beyond the influence of the mean glucose [15]. In patients with type 2 diabetes mellitus, fast plasma glucose (FPG) variability has been demonstrated to be a risk factor for DR independent of the mean FPG or HbA1c, particularly in cases of acute fluctuations and acute hyperglycemia $[16,17]$.

It is worthy to mention that there is no clear consensus on the gold-standard method for measuring GV in clinical practice; however, numerous variability indices have been proposed. Some of them can be obtained by downloading self-monitoring blood glucose (SMBG) data, making them available not only to diabetologists but also to patients. However, SMBG may not be appropriate for assessing GV because of the high number of determinations which are needed to evaluate the required parameters, such as standard deviation (SD), mean amplitude of glucose 
excursion (MAGE), and continuous overall net glycemic action (CONGA). Additionally, other indices, such as continuous glucose monitoring (CGM), may provide information on daily glucose fluctuations and can show how those numbers are affected by everyday activities and stress levels, although they are usually particularly complex [18]. Overall, the methods used for evaluating GV represent a critical issue and have several limitations, which should be taken into account when interpreting studies evaluating the association of $\mathrm{GV}$ with diabetes mellitus complications.

In conclusion, glycemic control remains an important factor for the presence and progression of DR. HbA1c seems to be an indicator which cannot demonstrate exactly the degree of glycemic control, while sudden variations of blood glucose may play an important role in DR; therefore, GV may be useful to predict DM complications, such as DR. Further studies are needed to scrutinize the impact of GV in DR progression and in response to various treatment modalities for DR.

\section{ACKNOWLEDGEMENTS}

Funding. No funding or sponsorship was received for publication of this article. All named authors meet the International Committee of Medical Journal Editors (ICMJE) criteria for authorship for this manuscript, take responsibility for the integrity of the work as a whole, and have given final approval for the version to be published.

Disclosures. Irini Chatziralli has nothing to disclose.

Compliance with Ethics Guidelines. This article is based on previously conducted studies and does not involve any new studies of human or animal subjects performed by the author.
Data Availability. Data sharing is not applicable to this article as no datasets were generated or analyzed during the current study.

Open Access. This article is distributed under the terms of the Creative Commons Attribution-NonCommercial 4.0 International License (http://creativecommons.org/licenses/ by-nc/4.0/), which permits any noncommercial use, distribution, and reproduction in any medium, provided you give appropriate credit to the original author(s) and the source, provide a link to the Creative Commons license, and indicate if changes were made.

\section{REFERENCES}

1. Yau JW, Rogers SL, Kawasaki R, et al. Global prevalence and major risk factors of diabetic retinopathy. Diabetes Care. 2012;35:556-64.

2. Leasher JL, Bourne RR, Flaxman SR, et al. Global estimates on the number of people blind or visually impaired by diabetic retinopathy: a meta-analysis from 1990 to 2010. Diabetes Care. 2016;39:1643-9.

3. Wat N, Wong RL, Wong IY. Associations between diabetic retinopathy and systemic risk factors. Hong Kong Med J. 2016;22:589-99.

4. Bansal AS, Khurana RN, Wieland MR, Wang PW, Van Everen SA, Tuomi L. Influence of glycosylated hemoglobin on the efficacy of ranibizumab for diabetic macular edema: a post hoc analysis of the RIDE/ RISE Trials. Ophthalmology. 2015;122:1573-9.

5. The Diabetes Control and Complications Trial Research Group. The relationship of glycemic exposure (HbA1c) to the risk of development and progression of retinopathy in the Diabetes Control and Complications Trial. Diabetes. 1995;44:968-83.

6. UK Prospective Diabetes Study (UKPDS) Group. Intensive blood-glucose control with sulphonylureas or insulin compared with conventional treatment and risk of complications in patients with type 2 diabetes (UKPDS 33). Lancet. 1998;352:837-53.

7. Diabetes Control and Complications Trial/Epidemiology of Diabetes Interventions and Complications Research Group, Lachin JM, Genuth S, Cleary P, Davis MD, Nathan DM. Retinopathy and nephropathy in patients with type 1 diabetes 
4 years after a trial of intensive therapy. $\mathrm{N}$ Engl J Med. 2000;342:381-389.

8. Chew EY, Ambrosius WT, Davis MD, et al. Effects of medical therapies on retinopathy progression in type 2 diabetes. N Engl J Med. 2010;363:233-44.

9. Hemmingsen B, Lund SS, Gluud C, et al. Targeting intensive glycaemic control versus targeting conventional glycaemic control for type 2 diabetes mellitus. Cochrane Database Syst Rev. 2013;11:CD008143.

10. American Diabetes Association. Glycemic targets. Diabetes Care. 2015;38(Suppl):S33-40.

11. Monnier L, Mas E, Ginet C, et al. Activation of oxidative stress by acute glucose fluctuations compared with sustained chronic hyperglycemia in patients with type 2 diabetes. JAMA. 2006;295:1681-7.

12. Cavalot F, Petrelli A, Traversa M, et al. Postprandial blood glucose is a stronger predictor of cardiovascular events than fasting blood glucose in type 2 diabetes mellitus, particularly in women: lessons from the San Luigi Gonzaga Diabetes Study. J Clin Endocrinol Metab. 2006;91:813-9.

13. Picconi F, Parravano M, Ylli D, et al. Retinal neurodegeneration in patients with type 1 diabetes mellitus: the role of glycemic variability. Acta Diabetol. 2017;54:489-97.

14. Virk SA, Donaghue KC, Cho YH, et al. Association between HbA1c variability and risk of microvascular complications in adolescents with type 1 diabetes. J Clin Endocrinol Metab. 2016;101:3257-63.

15. Lachin JM, Bebu I, Bergenstal RM, et al. Association of glycemic variability in type 1 diabetes with progression of microvascular outcomes in the Diabetes Control and Complications Trial. Diabetes Care. 2017;40:777-83.

16. Takao T, Ide $\mathrm{T}$, Yanagisawa $\mathrm{H}$, et al. The effect of fasting plasma glucose variability on the risk of retinopathy in type 2 diabetic patients: retrospective long-term follow-up. Diabetes Res Clin Pract. 2010;89:296-302.

17. Sartore G, Chilelli NC, Burlina S, Lapolla A. Association between glucose variability as assessed by continuous glucose monitoring (CGM) and diabetic retinopathy in type 1 and type 2 diabetes. Acta Diabetol. 2013;50:437-42.

18. Suh S, Kim JH. Glycemic variability: how do we measure it and why is it important? Diabetes Metab J. 2015;39:273-82. 